\section{NUCLEATION}

\section{That Factor $10^{17}$}

from our Molecular Physics Correspondent

THE strange controversy over the existence or non-existence of a factor $10^{17}$ in homogeneous nucleation rates seems to be dying down, leaving everybody not a little the wiser and more respectful of the subtleties of surface thermodynamics and the interplay of energy and entropy in very small systems. It is now nearly ten years since Lothe and Pound (J. Chem. Phys., 36, 2080; 1962) threw their carefully poised spanner into the works, arguing that the classical Becker-DöringVolmer-Zeldovitch treatment of the dynamics of droplet formation contained a false prescription for the handling of translational and rotational degrees of freedom when notionally dispersing a bulk liquid into nuclei of almost atomic dimensions. The sheer size of the discrepancy introduced-about $10^{17}$ in steady-state concentrations, but logarithmically less in terms of critical supersaturation-indicated that something more than a poor approximation was to blame, though the issue was confused by other dubious factors such as the use of a "surface tension" to write the free energy of even the smallest nuclei. Lothe and Pound, with somewhat more courage of conviction than modesty, indicted the classical theory on the particular count that it failed to represent the considerable entropy due to the Brownian motion of clusters, both translational and rotational. They then applied what seemed to be the obvious correction for this, obtained the factor $10^{17}$ for typical cases and accepted quite cheerfully that it destroyed agreement with the, admittedly not impressive, experimental data on critical supersaturations available at the time.

Since then, argument has ranged back and forth in the pages of the Journal of Chemical Physics and elsewhere, at times seeming only to deepen the confusion, more recently shifting, it seems, decisively, in favour of the chief antagonists of the Lothe-Pound position, H. Reiss and J. L. Katz of UCLA. Now two important constructive reviews of the subject have appeared (H. Reiss, J. Stat. Phys., 2, 83; 1970, and P. P. Wegener and J-Y. Parlange, Naturwissenschaften, 57, 525; 1970); the first makes a convincing attempt to say the last word on the translation-rotation paradox, the second reviews this in relation to more recent experimental results. The WegenerParlange article, incidentally, contains one of the best bibliographies in existence for this tortuous subject.

In this most recent critique Reiss meticulously reconstructs the basic equation for equilibrium concentrations of $\mathrm{N}$-atom clusters in a supersaturated gas using a very general formulation in terms of the classical configuration integral and postponing all details of the geometry and internal mechanics of the clusters for as long as possible. The only assumption at this stage, apart from classical mechanics and gross spatial homogeneity, is the existence of geometrically distinct clusters in the condensing gas, corresponding to certain factorizations of the total configuration integral. Reiss then defines the physical clusters by a new collective property, introducing the idea of a minimum sphere enclosing all atoms present to replace the centre of mass used as the locating property in his previous treatments. This seems to be the crucial step in solving the problem, because use of the minimal sphere both allows the centre of mass of the cluster to fluctuate realistically and at the same time leads to a definition of clusters in a form as close as possible to the nature of an actual physical drop. When these assumptions are carried through - a process considerably more intricate than can be rendered here-the result is no longer a factor of $10^{17}$ but a relatively harmless coefficient or order unity which is later shown to arise quite naturally as a correction for the fact that real clusters are not isolated in their internal motions but exist subject to their own vapour pressure.

Although there are still passages in Reiss's argument where sleight of hand could cover a fallacy, the final result is convincing even without the accumulating weight of experimental evidence with which it now brings the whole theory altogether more into line. This evidence, much of it arising from supersonic nozzle experiments, is also reviewed by Wegener and Parlange (loc. cit.) and is added to in the form of critical supersaturation calculations in a recent article by J. L. Katz (J. Stat. Phys., 2, 137; 1970) using the Reiss approach. Readers of these columns will also have noted recent activity in the cause of molecular beam cluster studies which seem likely to yield much more direct information about the properties of small growth nuclei in the near future (see Nature Physical Science, 229, 99; 1971).

Although the resolution of the "translation-rotation" paradox now seems to have gone against the original instigators, none of their critics has suggested that the flaw in Lothe and Pound's original argument was in any way superficial; on the contrary it is now generally recognized to have been embedded close to the heart of the central mystery - of how correctly to characterize a surface in micro-statistical terms while making a consistent guess (for it can still hardly be more) at the partition functions for the internal motions of small droplets. The considerable credit due to Lothe and Pound for throwing open the whole question is all the greater because, however dramatic the numerical factor involved, it was logical rather than numerical consistency which was at stake. At the same time Reiss and his collaborators can be well satisfied at having, so to speak, cleared the decks of a nasty theoretical problem in time for the next round of experiments.

\title{
Anomalous Water and Silicic Acid
}

ON page 31 of this issue of Nature, Barnes et al. clearly state their belief that the properties of anomalous water are no more than the sum of the properties of the impurities present and that anomalous water as such probably does not exist. Some support is given to this contention by the work of Adams et al., an account of which appears in next Monday's Nature Physical Science; they claim that the "anomalous water" produced in their experiment is chiefly a silicic acid solution.

Adams and his colleagues have made use of the high surface to volume ratio of porous glass to create the same environment for the production of anomalous water as other workers who have used Pyrex capillaries, for example. They took a number of precautions to reduce contamination which included burning off organic impurities in the porous glass at $550^{\circ} \mathrm{C}$ in a stream of oxygen and the use of greaseless Tefion valves throughout the apparatus. They also used a quartz iodide lamp to irradiate the porous glass cell on the grounds that strong irradiation would enhance the surface reactions which have been generally thought necessary for the successful production of anoma- lous water. Adams et al. found that the liquid which condensed after passing through the walls of the porous glass container showed many of the properties of the anomalous water reported last year by Deryagin (Sci. Amer., 223, 52; 1970).

These properties are not spelt out by Adams et al., but presumably they include high values for molecular weight and boiling point, for example. Desiccation of the liquid samples revealed solid bodies which contained between 10 and 30 per cent of silicon and about 5 per cent sodium by weight according to electron microprobe analysis. They point out that this is consistent with the work of Morariu et al. (Nature, 227, 373; 1970), who found similar bodies in samples of anomalous water and conjectured that these solids had condensed from silicic acid solution; unfortunately, they performed no chemical analyses to support their hypothesis. Adams et al. suggest that the anomalous water which they produced is almost certainly a silicic acid solution and this goes a long way towards establishing experimentally that the chief characteristic of anomalous water may be that it does not exist. 